\title{
OLD WINE IN A NEW BOTTLE - ENTEROCOCCAL URINARY TRACT INFECTION IN NOSOCOMIAL SETUP
}

Indrani Bhattacharyya ${ }^{1}$, Jayashree Konar², Sanjeev Das ${ }^{3}$, Pratip Kumar Kundu'4, Md. Samidul Hoque ${ }^{5}$

\section{HOW TO CITE THIS ARTICLE:}

Indrani Bhattacharyya, Jayashree Konar, Sanjeev D as, Pratip Kumar Kundu, Md. Samidul Hoque. "Old wine in a new bottle - enterococcal urinary tract infection in nosocomial setup". Journal of Evolution of Medical and Dental Sciences 2013; Vol. 2, Issue 43, Octo ber 28; Page: 82 77-8283.

ABSTRACT: Enterococcal UTI is a well-known source of fatal bacteraemia \& endocarditis. OBJECTIVE: Identification of different species of Enterococci causing nosocomial urinary tract infection in a tertiary care hospital along with antibiotic susceptibility pattern. METHODS: Admitted patients developing symptoms of UTI atleast after 48 hours of admission were included in this study over a period from January-June 2013.Urine samples collected in appropriate sterile manner were screened for pus cells and bacteria followed by speciation according to Facklam-Collins scheme. Enterococcal isolates were preserved in CTA until tested by VITEK2 (bioMerieux) with AES to confirm the speciation. Antibiogram was performed by disk diffusion method (modified Kirby-Bauer technique) on Muller-Hinton agar and blood agar media. MIC of the tested antibiotics was detected by VITEK 2 with AES which provides accurate "fingerprint" recognition of bacterial resistance. RESULTS: Out of total 187 urine samples, 34 were culture positive (18\%) of which Enterococci were isolated in 9 cases (26.4\%); Enterococcus faecalis \& Enterococcus faecium four each along with one Enterococcus gallinarum strain. Isolated Enterococcus gallinarum and all isolated Enterococcus faecalis were sensitive to Penicillin group of drugs, although all isolated Enterococcus faecium were resistant to them. None of the Enterococcal isolates produced $\beta$ lactamase. In isolated Enterococcus gallinarum and one Enterococcus faecalis isolate, MIC value of Ampicillin was double that of Benzyl-penicillin. Isolated Enterococcus gallinarum was the only Vancomycin resistant Enterococcus strain. According to VITEK2 AEC, it was of vanA type with MIC $\geq 32 \mu \mathrm{g} / \mathrm{ml}$. All four Enterococcus faecalis isolates were resistant to Quinupristin \& Dalfopristin whereas isolated Enterococcus faecium were sensitive to those antimicrobials. All of the isolates were sensitive to Nitrofurantoin except one Enterococcus faecium isolate (MIC value $64 \mu \mathrm{g} / \mathrm{ml}$ ) and Enterococcus gallinarum isolate (MIC value $64 \mu \mathrm{g} / \mathrm{ml}$ ), both of which were intermediately susceptible to the drug. CONCLUSION: Four Enterococcal isolates were susceptible to Ampicillin and Aminoglycosides (high level synergy) suggesting use of combination therapy of Ampicillin and Aminoglycosides for treatment. Vancomycin and Linezolid are the only available drugs for treatment of isolates resistant to both or any one of the drugs Ampicillin and Aminoglycosides (high level synergy). To treat VRE, Linezolid may be prescribed.

INTRODUCTION: Enterococci are Gram positive oval shaped cocci that are typically arranged in pairs \& short chains and at an angle to each other. They are facultative anaerobe and homofermentative. They are nonmotile (except E gallinarum and E casseliflavus), non capsulated organism nonhemolytic usually but sometimes show alpha or beta hemolysis ${ }^{1,2}$.

BACKGROUND: Enterococci are the second most common cause of nosocomial urinary tract infection in Western hemisphere ${ }^{3}$. 
Enterococcus faecalis is the most common isolate being associated with $80-90 \%$ of human Enterococcal infection, Enterococcus faecium ranks second and is isolated from 10-15\% of infections. Other species are infrequently isolated from clinical specimens ${ }^{4}$.

In vitro, Enterococci have penicillin MICs 10 to 100 fold higher than that of Streptococci5.

In the US, $>90 \%$ of isolated Enterococcus faecium are resistant to Ampicillin whereas resistance to Ampicillin is much less common in Enterococcus faecalis (4\%) $6,7,8$.

Undiagnosed and untreated Enterococcal UTI is a well known source of fatal Enterococcal bacteraemia \& endocarditis especially in immunocompromised patients and nosocomial set up6, 7, 8. Objective of this study was to identify different species of Enterococci causing nosocomial urinary tract infection in a tertiary care hospital. In addition, antibiotic susceptibility pattern of the isolated strains was also studied because every microbiological diagnosis should ultimately aim at appropriate treatment for reduction of morbidity\& mortality.

METHODS: The study was carried out in a tertiary care set up in West-Bengal over a period of six months from January 2013 to June 2013.

Study population: Patients admitted in a tertiary care hospital, developing symptoms of UTI atleast after 48 hours of admission.

UTI cases with established non bacterial aetiology were not included in the study.

Study design: Urine samples collected in appropriate sterile manner were screened for pus cells and bacteria. This was followed by plating on Mac-conkey's agar media (differential \& partially selective media: aids in isolation of gram negative isolates, Enterococci give small pin point lactose fermenting translucent colonies), Blood agar (enriched media for Enterococci isolation, Enterococci produce mostly nonhemolytic colonies in sheep blood agar). Inoculated plates were incubated overnight at $37^{\circ} \mathrm{c}$. Discrete colonies were further studied by Gram staining, tests for motility, battery of biochemical test according to Facklam-Collins scheme (Facklam and Collins, 1989) for Enterococcal speciation7. Isolated Enterococcus strains were preserved in CTA (Cystine Tryptic Agar) media at $4^{0} \mathrm{c}$ until tested by VITEK 2Microbial identification system (bioMerieux) with "Advanced Expert System" (AES) to confirm the speciation.

Antibiogram was performed by disk diffusion method (modified Kirby-Bauer technique) on Muller-Hinton agar and blood agar media. MIC (Minimum Inhibitory Concentration) values of the tested antibiotics were detected by VITEK 2 with "Advanced Expert System" (AES) as per CLSI guideline. With its ability to provide accurate "fingerprint" recognition of bacterial resistance mechanisms and phenotypes, the Advanced Expert System (AES) is a critical component of VITEK 2 technology ${ }^{9}$.Results were analysed according to standard statistical method.

RESULTS: Out of total 187 urine samples from selected patients, 34were culture positive (18\%). Out of 34 culture positive cases, Enterococci were isolated in 9 cases (26.4\%). Enterococcus faecalis \& Enterococcus faecium were found in equal number of cases (four each). One Enterococcus gallinarum strain was also isolated. However Escherichia coli were isolated in 19 cases (55\%).

None of the Enterococcal isolates produced $\beta$-lactamase and all of them were sensitive Tigecycline\& resistant to Polymyxin-B. 
One interesting finding in this study was that isolated Enterococcus gallinarum and all four isolated Enterococcus faecalis were sensitive to both Benzyl penicillin and Ampicillin although all isolated Enterococcus faecium were resistant to both of the drugs.

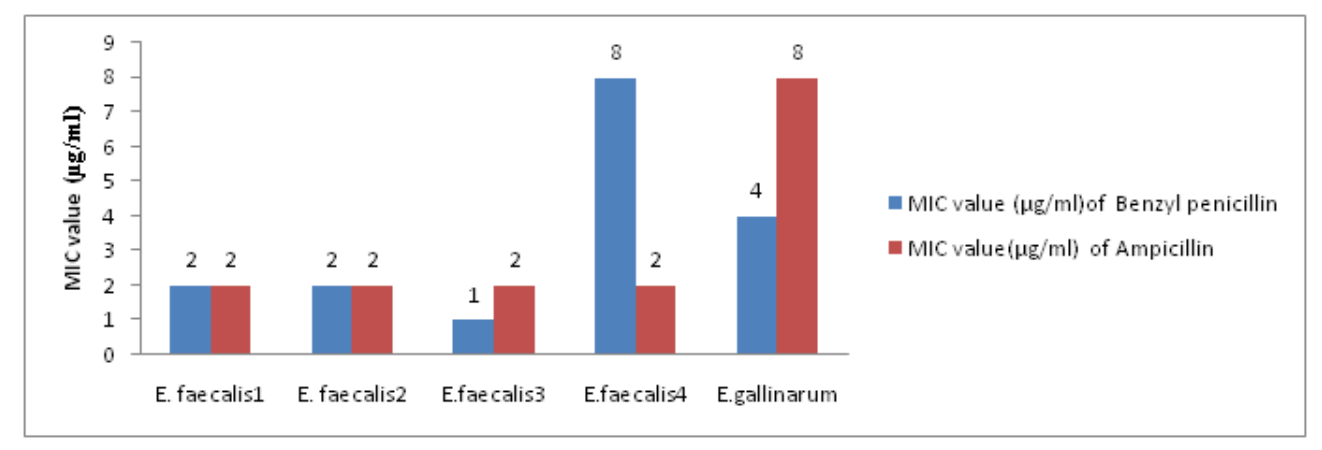

Fig-1: Bar diagram shows MIC values of $\beta$-lactam antimicrobials to which, isolated Enterococci were susceptible. In isolated Enterococcus gallinarum and the third Enterococcus faecalis isolate, MIC value of Ampicillin was double that of Benzyl penicillin.

All isolates of Enterococcus faecalis and Enterococcus faecium were sensitive to Vancomycin and Linezolid but Enterococcus gallinarum isolate inspite of being Benzyl penicillin sensitive, was Vancomycin resistant (MIC value is $\geq 32 \mu \mathrm{g} / \mathrm{ml}$ ). In addition, the resistance pattern was van-A type (fingerprint was identified by VITEK 2Advanced Expert System).

All of the Enterococcus faecalis isolates were resistant to Quinupristin \& Dalfopristin whereas isolated Enterococcus faecium were sensitive to those antimicrobials.

All of the isolates were sensitive to Nitrofurantoin except one Enterococcus faecium isolate (MIC value $=64 \mu \mathrm{g} / \mathrm{ml}$ ) and the only Enterococcus gallinarum isolate (MIC value $=64 \mu \mathrm{g} / \mathrm{ml}$ ), both of which were intermediately susceptible to the drug.

Resistance pattern of other Antimicrobials are depicted in the following bar diagram:

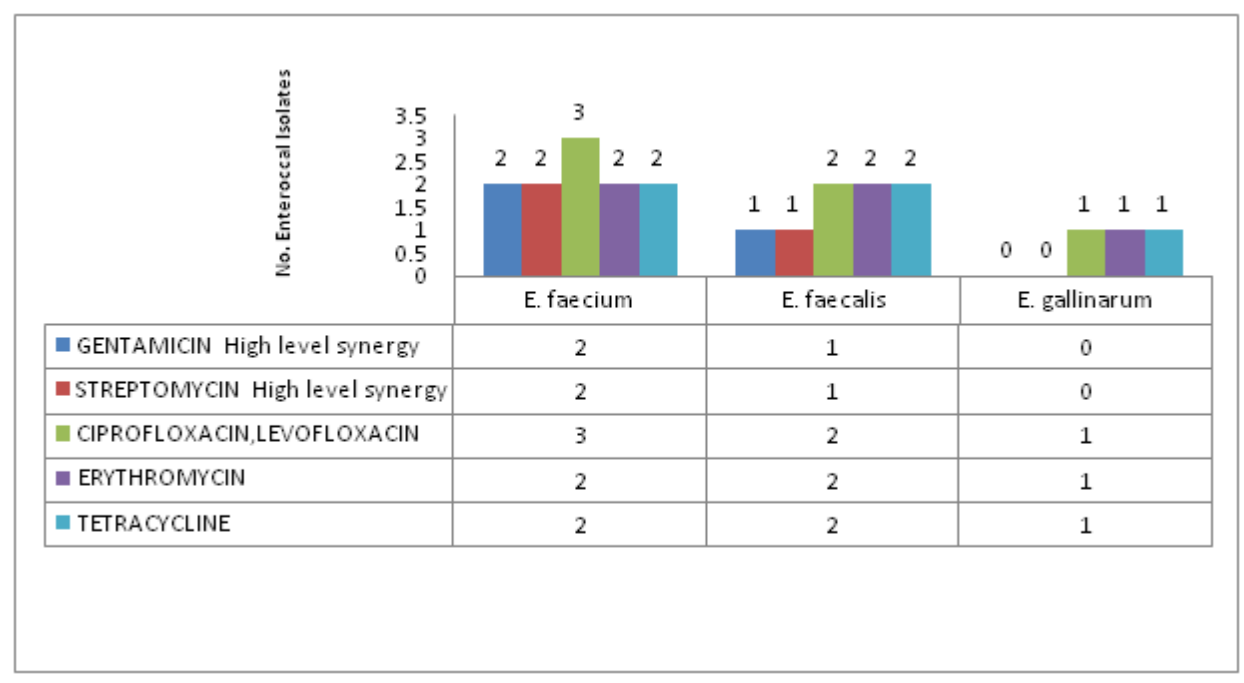

Fig-2: Bar diagram shows that Enterococcus faecium isolates were more resistant to various antimicrobials than Enterococcus faecalis. 
DISCUSSION: The overall occurrence of Enterococcal infection varies across continents, countries and also within hospitals. In India, the occurrence varies from $1 \%$ to $36 \%$. In 2003, Karmakar et al carried out a study in Mumbai in which the isolation rate of Enterococci from urine samples was $10.28 \%{ }^{10}$. In March 2006, Kaur et al reported an Enterococcal isolation rate of $33 \%$ from urine samples in Haryana11.In 2008, Agarwal et al in Lucknow showed, an isolation rate of $1.46 \%$ in diverse clinical samples ${ }^{12}$.

Our study showed $4.8 \%$ of Enterococcal isolation ( 9 out of 187 collected samples) although it comprised of $26.4 \%$ of total culture isolates ( 9 out of 34), which is higher than the finding of Agarwal etal but lower than that of other workers.

In contrast to study of some other workers such as Duo et al (Kuwait, 2002) \& Ford et al (UK, 1994), Enterococcus faecalis was not the most prevalent isolate. Enterococcus faecalis and Enterococcus faecium were isolated in equal number (four each) in our study.

Antimicrobial susceptibility pattern revealed the surprising fact that three Enterococcus faecalis and Enterococcus gallinarum isolates were sensitive to $\beta$-lactam antimicrobials (Benzyl penicillin and Ampicillin) and Aminoglycosides (Gentamicin and Streptomycin high level synergy) suggesting use of combination therapy of $\beta$-lactams and Aminoglycosides for treatment. Out of four isolated Enterococcus faecium, two were resistant to both Ampicillin and Aminoglycosides (high level synergy), whereas, the other two were resistant to $\beta$-lactam antibiotics (Benzyl penicillin and Ampicillin) but sensitive to Aminoglycosides (high level synergy). One Enterococcus faecalis isolate was sensitive to Benzyl penicillin and Ampicillin but resistant to Aminoglycosides (high level synergy). In these cases, above said combination therapy seemed to be ineffective.

Fluoroquinolones (66.7\% resistance) and Macrolides (55.6\% resistance) should not be used empirically for treatment of Enterococcal infection.

Out of total nine isolated Enterococci, five were multidrug resistant (55.55\%). Three out of four isolated Enterococcus faecium (75\%) and one out of four isolated Enterococcus faecalis $(33.33 \%)$ along with the isolated Enterococcus gallinarum comprised this multidrug resistant group. As no Vancomycin and/or Linezolid resistance was present, those two drugs were the only choice for treatment of those multidrug resistant Enterococci.

Out of nine Enterococcal isolates only one (Enterococcus gallinarum) was Vancomycin resistant (MIC value $\geq 32 \mu \mathrm{g} / \mathrm{ml}$ ) \& this resistance pattern was diagnosed as vanA type of resistance (by VITEK 2 system).

Karmakar et al (Mumbai, 2003) found more than 20\% of Enterococcal isolates were VRE, whereas Agarwal et al (Lucknow, 2008) found nearly 2\% of isolates to be VRE. In our study, 11.11\% of isolated Enterococcus was VRE. Similar to other studies, in our study also, VRE isolate was susceptible to Linezolid in addition to that, surprisingly enough it was also sensitive to $\beta$-lactams and aminoglycosides (high level synergy) but it was resistant to Fluoroquinolones, Macrolides and Tetracyclines.

Isolated VRE was intermediately susceptible to Nitrofurantoin (MIC=64 $\mathrm{g} / \mathrm{ml}$ ). One Enterococcus faecium isolate (Vancomycin sensitive) was also intermediately susceptible to Nitrofurantoin (MIC=64 $\mathrm{mg} / \mathrm{ml}$ ). Rest of the seven isolates was sensitive to Nitrofurantoin. Enterococcal susceptibility pattern to Nitrofurantoin is inconsistently reported by other workers. 
In contrast to a study done by G. Werner et $\mathrm{al}^{13}$,in our study we have found no DalfopristinQuinupristin resistance in Enterococcus faecium whereas all four Enterococcus faecalis isolates were resistant to Streptogramin antibiotics (Dalfopristin-Quinupristin).

While looking through our laboratory log books retrospectively, certain numerical data glared at us. Between January 2012-June2012, Enterococci were responsible for 7.5\% of nosocomial UTI. The corresponding data between July 2012 and December 2012 were $11.34 \%$, in the study period, the percentage was $26.4 \%$ !! Thus there is a progressive increase in nosocomial UTI caused by Enterococci. This led us to opt for the automated system (VITEK 2 Microbial identification system with Advanced Expert System). Inspite of being costly the use of VITEK 2 is justified because rapid \& accurate results allow clinicians to discontinue empiric therapy and prescribe targeted therapy, resulting in improved patient outcome and enhanced antibiotic stewardship ${ }^{9}$. In addition, Advanced Expert System (AES) provide us "fingerprint" recognition of vanA gene present in Vancomycin resistant Enterococcus gallinarum. Further extension of this study with larger number of cases may help us to bring about a calculated and logical revision in the antibiotic policy of Enterococcal urinary tract infection in nosocomial setup.

\section{REFERENCES:}

1. Washington W, StephenA, Willium J, Elmir K, Gary W, Paul S, Gail. Gram positive Cocci Part II: Streptococci, Enterococci and the Streptococcus-like Bacteria in Koneman's color Atlas and text book of diagnostic Microbiology $6^{\text {th }}$ ed. Lippincolt, William \& Wilkins, London, 2006; 674-675.

2. Hierarchial classification of clinically significant bacteria in Bergy's Manual of SystemicBacteriology,2nd edition in Koneman's color Atlas and text book of diagnostic Microbiology $6^{\text {th }}$ ed. Lippincott, William \& Wilkins,London,2006;174.

3. Felmingham D, Wilson PR, Quintana AI, Gruneberg RN, Enterococcus species in urinary tract infection. Clin Infect Dis 1992:15:295-305.

4. Ruoff KL, de la MZ L, Murtaugh MJ, et al. Species identification of Enterococci from clinical specimens. J Clin Microbiol 1990; 28:435-437.

5. Washington W, Stephen A, Willium J, Elmir K, Gary W, Paul S,Gail. Gram positive Cocci Part II: Streptococci, Enterococci and the Streptococcus-like Bacteria in Koneman's color Atlas and text book of diagnostic Microbiology $6^{\text {th }}$ ed. Lippincott, William \& Wilkins, London, 2006; 700-701.

6. Arias CA, Murray BE: Enterococcus species, Streptococcus bovis group and Leuconostoc species, in Principles and Practice of Infectious Diseases, 7th ed, GL Mandell et al. Philadelphia, Churchill Livingstone Elsevier, 2010, pp 2643-2654.

7. Facklam RR et al: History, taxonomy, biochemical characteristics and antibiotic susceptibility testing of enterococci, in The Enterococci: Pathogenesis, Molecular Biology and Antibiotic Resistance, MS Gilmore (ed). Washington, DC, ASM Press, 2002, pp 1-54.

8. Willems RJ, van Schaik W: Transition of Enterococcus faecium from commensal organism to nosocomial pathogen. Future Microbiol 4:1125, 2009 [PMID: 19895216].

9. Steward, Christine D., Raney, Patti M., Morrell, Allison K., Williams, Portia P., McDougal, Linda K., Jevitt, Laura, McGowan, John E., Jr., Tenover, Fred C. Testing for Induction of 
Clindamycin Resistance in Erythromycin-Resistant Isolates of Staphylococcus aureus. J. Clin. Microbiol. 2005 43: 1716-1721.

10. MG Karmakar, Edwin S Greshom and P R Mehta. Enterococcal infection with special reference to phenotypic characterisation and drug resistance. Indian J Med. Res.2004; 119 (Supple)22-25.

11. Narinder Kaur, Uma Chaudhury, Ritu Aggrwal. Emergence of VRE and their antimicrobial sensitivity pattern in a tertiary care teaching hospital. R.C i.med. biol., 2009; 8:281-286.

12. J. Agarwal, R Kalyan, M Singh, High Level Aminoglycoside resistance and $\beta$-lactamase production IN Enterococci at a tertiary care hospital of India; J pn. J Infect Dis2009; 62:158-159.

13. G Werner, I Klare, H Heier, KH Hinz, G Böhme, $M$ Wendt, and $W$ Witte. Quinupristin/Dalfopristin-Resistant Enterococci of the sat A (vatD) and sat G (vat E) Genotypes from Different Ecological Origins in Germany: Microbial Drug Resistance; Spring 2000, 6(1): 37-47.

\begin{tabular}{|c|c|c|c|c|c|c|}
\hline Sl.No. & Lab No. & Lot No. & Bio No. & Date & Time (IST) & Result \\
\hline 1 & Lab ID $: 2$ & - & 116012365773671 & $24 / 02 / 2013$ & $08: 14$ & Enterococcus faecalis \\
\hline 2 & Lab ID :3 & - & 010047065753671 & $24 / 02 / 2013$ & $12: 06$ & Enterococcus faecium \\
\hline 3 & Lab ID : 5 & - & 116002665773671 & $24 / 02 / 2013$ & $12: 07$ & Enterococcus faecalis \\
\hline 4 & Lab ID : 6 & - & 030047065751671 & $24 / 02 / 2013$ & $12: 07$ & Enterococcus faecium \\
\hline 5 & Lab ID : 13 & - & 114002761773671 & $24 / 02 / 2013$ & $12: 08$ & Enterococcus faecalis \\
\hline 6 & Lab ID $: 14$ & - & 114012761773671 & $24 / 02 / 2013$ & $12: 09$ & Enterococcus faecalis \\
\hline 7 & Lab ID : 20 & - & 514412367773661 & $24 / 02 / 2013$ & $12: 09$ & Enterococcus gallinarum \\
\hline 8 & U-253-1 & 242269410 & 034003265771771 & $04 / 03 / 2013$ & $18: 34$ & Enterococcus faecium \\
\hline 9 & U-246-1 & 242269410 & 030002065713651 & $05 / 03 / 2013$ & $20: 37$ & Enterococcus faecium \\
\hline
\end{tabular}

\section{ABBREVIATIONS:}

AES- Advanced Expert System.

CLSI- Clinical laboratory standard institute.

CTA- Cystine Tryptic Agar.

HLS- High level synergy.

MIC- Minimum inhibitory concentration.

UTI- Urinary tract infection.

VRE- Vancomycin resistant Enterococcus. 


\section{AUTHORS:}

1. Indrani Bhattacharyya

2. Jayashree Konar

3. Sanjeev Das

4. Pratip Kum ar Kundu

5. Md. Samidul Hoque

\section{PARTI CULARS OF CONTRIBUTORS:}

1. Assistant Professor, Department of Microbiology, Calcutta school of Tropical Medicine, Kolkata.

2. PGT, Department of Microbiology, Calcutta school of Tropical Medicine, Kolkata.

3. Assistant Professor, Department of Microbiology, Murshidabad Medical College, Murshidabad.
4. Professor, Department of Microbiology, Calcutta scho ol of Tropical Medicine, Kolkata.

5. Lab Tech, Department of Microbiology, Calcutta scho ol of Tropical Medicine, Kolkata.

\section{NAME ADDRESS EMAIL ID OF THE CORRESPONDING AUTHOR:}

Dr. Indrani Bhattacharyya,

37J/1D, Raja Manindra Road, Kolkata - 700037.

Email - indranichaudhuri@yahoo.com

Date of Submission: 05/10/2013.

Date of Peer Review: 07/10/2013.

Date of Acceptance: 10/10/2013.

Date of Publishing: 23/10/2013 